\title{
Amorphous and crystalline phase interaction during the Brill transition in nylon 66
}

\author{
Y. Wolanov, A. Y. Feldman, H. Harel*, G. Marom \\ Casali Institute of Applied Chemistry, The Institute of Chemistry, The Hebrew University of Jerusalem, 91904 Jerusalem, \\ Israel
}

Received 1 April 2009; accepted in revised form 4 May 2009

\begin{abstract}
A prominent $\alpha^{\prime}$ process in specifically treated nylon 66 and microcomposite samples is identified by dynamic mechanical analysis and proposed to be an amorphous phase counterpart of the Brill transition identified by synchrotron wide-angle X-ray diffraction (WAXD). It is suggested that this $\alpha^{\prime}$ process, which marks a critical free volume change and an onset of segmental chain movement in the amorphous phase, precedes and prompts the Brill transition in the crystalline phase.
\end{abstract}

Keywords: thermal properties, Brill transition, $\alpha$ relaxation

\section{Introduction}

The existence of certain associations between second and first order transitions in polymers is by now a proven fact. Many examples exist in the literature, of which the most prominent is the fact that a constant ratio exists between the glass transition temperature in the amorphous phase and the melting point. By and large, for polymers that are free from side groups (so called symmetrical) this ratio $\left(T_{g} / T_{m}\right)$ is $\sim 0.5$, whereas for most of the common vinyl, vinylidene and condensation polymers the ratio is $\sim 0.66$ [1]. Another example comes from the solid state crystallization process where the crystallization temperature $T_{c}$ is directly related to $T_{g}$. In this particular example, the relation between the second to the first order transition is causative, wherein the occurrence of the latter is consequent to the first, resulting from the necessity of sufficient free volume, attained at $T_{g}$, to allow chain segmental movement and crystallization. Two examples of our own studies are shown here. The first, describes an investigation of the effect of the cooling rate of a quenching process on the structure of glycolic acid (GA) and lactic acid (LA) [2]. The second, investigated the effect of quenching on the degree of crystallininity in carbon fibre-reinforced poly(ether ether ketone) (PEEK) composites. [3].

The bilateral phase interaction can also work in the opposite direction so that structural changes in the crystalline phase influence the morphology of the amorphous one. An appropriate example from our own research is of high density polyethylene (HDPE), which in its pristine form exhibits a single second order transition, namely, $T_{g}$. However, after undergoing a certain solid state crystallization stage, which results in crystalline restructuring, the same HDPE exhibits an untypically immense $\beta$ transition in the amorphous phase $[4,5]$.

In the present study we deal with yet another first order transition, namely the Brill transition in polyamides (e.g., nylon 66), for which a counterpart transition in the amorphous phase is explored. 
The Brill transition, discovered in 1942 [6], is a sharp reversible polymorphic phase transition, from the triclinic to the pseudo-hexagonal structure at $162^{\circ} \mathrm{C}$. It is preceded by gradual crystallographic changes, occurring over a relatively wide temperature range and entailing dimensional changes of the triclinic crystallographic unit cell, wherein the room temperature spacings of $0.44 \mathrm{~nm}\left(d_{100}\right)$ and $0.37 \mathrm{~nm}\left(d_{010 / 110}\right)$ are shifted to lower and higher values, respectively. At the transition point the two diffraction maxima are replaced by one with a spacing of $0.42 \mathrm{~nm}$ [6-8]. Over the years since its discovery, the Brill transition has been studied for various types of even-even and odd-even polyamides [9-13] and for different crystallization conditions [10].

In a recent study on the Brill transition in transcrystalline nylon 66 we pointed out that the explanation for the effect of the crystalline structure on this first order transition must be based on the existence of reciprocal interactions between the crystalline and amorphous phases [14], which corresponded to the finding that structural changes within the crystalline domains in the lamellae (as in the Brill transition) are accompanied by changes in the packing of the amorphous chain segments outside the lamellae [15]. According to the scientific literature, nylon 66 exhibits three loss peaks, labeled $\alpha, \beta$ and $\gamma$ (see e.g., [16-18]), representing different processes in the amorphous phase. Perhaps the only reference to an additional peak (shoulder) was made by Takayanagi in his study of nylon 6 [19]. Because this shoulder was most prominent for specimens with maximum crystallinity it was ascribed to a relaxation process involving the crystalline regions and was attributed to a crystal structure change from monoclinic to hexagonal [20]. This is taken by us as strong evidence for the assumption that the Brill transition in the crystalline phase is preceded or even triggered by a second order process in the amorphous phase. Accordingly, the present study has been undertaken in order to identify such a process that can be correlated with the Brill transition.

\section{Experimental}

\subsection{Materials}

Nylon 66 pellets were obtained from Nilit, Israel $\left(M_{w}=325000\right.$ and $\left.M_{n}=16800\right)$.
Aramid fibers, Kevlar 49 (Du-Pont 1420 denier yarn) were used as reinforcement.

\subsection{Sample preparation}

Films of approximately $100 \mu \mathrm{m}$ thickness were prepared by pressing the pellets at $290^{\circ} \mathrm{C}$ under minimal pressure for $20 \mathrm{~min}$ in a hydraulic press (Carver 2518), followed by isothermal treatment at $250^{\circ} \mathrm{C}$ for $3-8 \mathrm{~h}$ in the press followed by slow cooling.

Unidirectional microcomposites of nylon 66 reinforced by aramid fibers and displaying a transcrystalline layer (tcl) were prepared according to the procedure published in [21]. The condition ranges were similar to those used for the films. A fully developed tcl was observed with $3 \mathrm{~h}$ isothermal treatment at $250^{\circ} \mathrm{C}$ followed by ice-water quenching.

\subsection{In situ WAXD}

Synchrotron microbeam wide-angle X-ray diffraction (WAXD) measurements were performed at the European Synchrotron Radiation Facility (ESRF) on the Materials Science Beamline (ID-11). The X-ray microbeam was monochromated at a wavelength of $\lambda=0.4876 \AA$ and collimated to dimension $2 \mu \mathrm{m}$ (vertical) by $4 \mu \mathrm{m}$ (horizontal). The exposure time for each sample varied from 20 to $30 \mathrm{~s}$. Samples were inserted into $1 \mathrm{~mm}$ diameter Li-glass capillaries and mounted inside a hot stage (Linkam Scientific Instruments, THMS600, Waterfield, UK). The distance between the sample and the detector was set at approximately $143 \mathrm{~mm}$. Each sample was scanned during heating at different heating rates, from 25 to $400^{\circ} \mathrm{C}$. Data were taken at 5 or $10^{\circ} \mathrm{C}$ intervals. The one-dimensional diffraction profiles were calculated from the two-dimensional X-ray diffraction patterns using the image analysis programs Fit2D (ESRF, Dr. Hammersley).

\subsection{Dynamic mechanical analysis (DMA)}

Thermomechanical properties were investigated by dynamic mechanical analysis. A TA Instruments (New Castle, DE) DMA, model 983 was used to measure the transition temperatures. All samples (of the dimensions $10 \times 6 \times 0.1 \mathrm{~mm}^{3}$ ) were tested at fixed frequency mode under shear stress. The tem- 
perature was scanned from $25-220^{\circ} \mathrm{C}$ at a heating rate of $5^{\circ} \mathrm{C} / \mathrm{min}$, recording simultaneously the real and the imaginary components of the shear modulus and $\tan \delta$. The microcomposite specimens were loaded along the fiber axis.

\section{Results and discussion}

The essential changes that occur at the Brill transition are expressed clearly by 2D WAXD images below and above the transition temperature $T_{B}$, an example of which is presented in Figure 1 (scanned at a heating rate of $15^{\circ} \mathrm{C} / \mathrm{min}$ ). The typical features in the diffraction patterns are the clear 100 and 010/110 diffraction rings of the triclinic phase below $T_{B}$ and the 'shifted 100' diffraction ring of the pseudo-hexagonal phase above it. For the detection of the Brill transition, a series of such 2D diffraction patterns obtained during synchrotron WAXD in situ measurements can then be processed to produce 3D representations of the diffraction profiles as a function of temperature. The results for the nylon 66 films isothermally treated at $250^{\circ} \mathrm{C}$ are presented in Figure 2 for heating in the temperature range from 25 to $220^{\circ} \mathrm{C}$. For this particular material the Brill transition temperature is determined to be $T_{B}=175^{\circ} \mathrm{C}$.

The values of $T_{B}$ can be determined by measuring the $d$ spacings for the 100 and $010 / 110$ diffraction maxima, shown in Figure 2, and plotting them as a function of temperature as presented in Figure $3 ; T_{B}$ is the intercept of the two curves. It is noted that the point at $175^{\circ} \mathrm{C}$ (in Figure 3) represents the merging

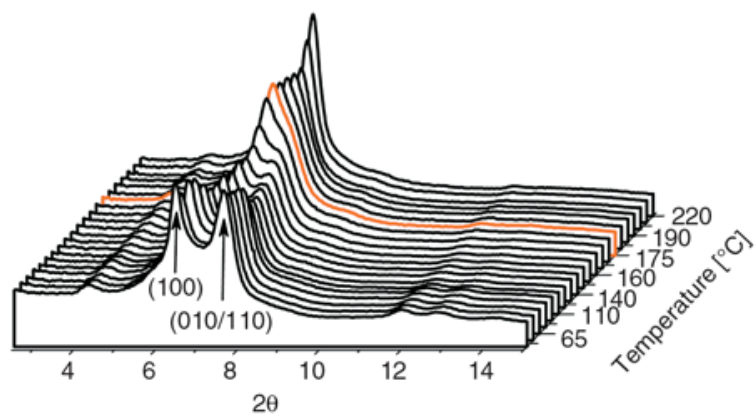

Figure 2. Three-dimensional representation of X-ray diffraction profiles of a nylon 66 film during heating at a rate of $15^{\circ} \mathrm{C} / \mathrm{min}$ from 25 to $220^{\circ} \mathrm{C}$

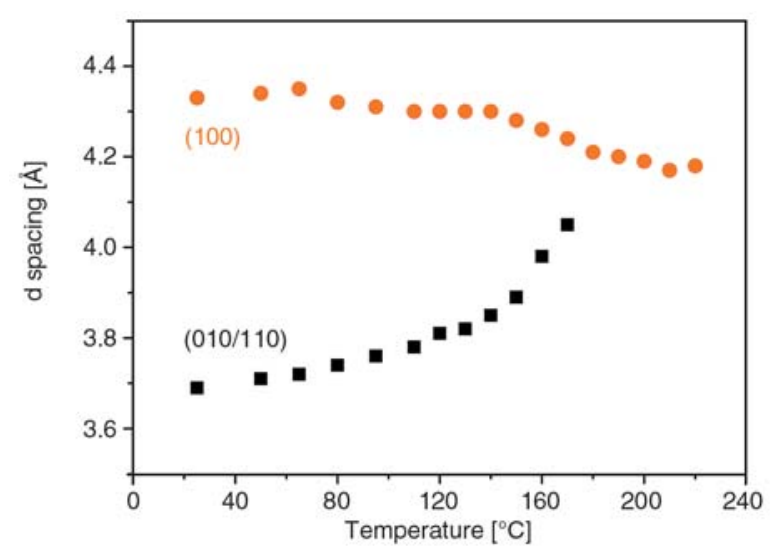

Figure 3. Temperature dependence of the $d$ spacings of the 100 and $010 / 110$ diffraction maxima of a nylon 66 film

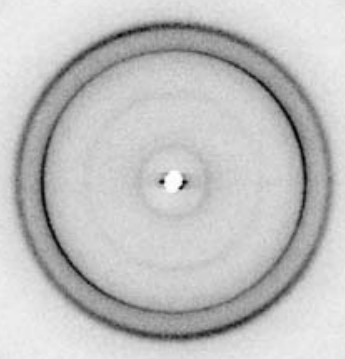

a)

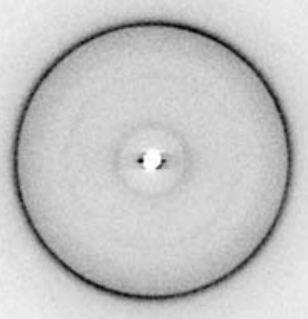

b)

Figure 1. 2D WAXD images of a nylon 66 film, at (a) 65 and (b) $210^{\circ} \mathrm{C}$, below and above the Brill temperature 
of the $010 / 110$ and $100 d$ spacings. Alternatively, $T_{B}$ can be determined by plotting the arithmetic difference between these two $d$ spacings of the triclinic cell (designated the index of chain packing, ICP) against temperature and extrapolating the linear region to ICP equals zero (not shown here) [14]. Looking at Figure 3 again, it is apparent that whereas the change in the $d$ spacings with temperature of the 100 diffraction is relatively gradual and small (with a few minor fluctuations), that of the 010/110 diffraction is characterized by a two step behavior. Over the first step, at the lower temperature range, the spacing change with temperature is minimal. Then at around $130^{\circ} \mathrm{C}$ a marked change is observed, followed by a steeper increase from approximately 3.8 to $4.2 \AA$ at $T_{B}$.

Assuming that this sharp change marks a critical free volume change and an onset of segmental chain movement in the amorphous phase, it was reasonable to assume that if a second order counterpart transition of the Brill transition existed it should be found at around this temperature. Accordingly, a range of nylon 66 samples and microcomposites [22] was prepared under different crystallization conditions and then subjected to DMA measurements. The DMA traces by and large revealed the typical three transitions $\alpha, \beta$ and $\gamma$, however, in a small number of specific cases, limited only to samples with a history of a long crystallization treatment, a clear $\alpha^{\prime}$ peak was observed. Particularly, a prominent $\alpha^{\prime}$ peak was observed for the microcomposites. It is well known that aramid fiber reinforced nylon 66 composites transcrystallize in such a way that a transcrystalline layer invades the whole space between the fibers and replaces most of the bulk crystallinity [23]. The amplification of the $\alpha^{\prime}$ peak can then be associated with the transcrystalline structure, similar to the $\beta$ peak in HDPE [5].

Two examples of samples that exhibit an apparent $\alpha^{\prime}$ peak are shown in Figure 4. It is noted that the microcomposite in Figure $4 \mathrm{~b}$ exhibits a clear split $\alpha$ process, wherein the glass transition is followed by a separate smaller process. The $\alpha^{\prime}$ process reaches its maximum at $110^{\circ} \mathrm{C}$ for the pure nylon 66 and at $125^{\circ} \mathrm{C}$ for the microcomposite, which correspond to the sharp tilt at around $130^{\circ} \mathrm{C}$ observed in the $d$ spacings for the $010 / 110$ diffraction maxima of the triclinic crystalline phase in Figure 3. This is considered as strong evidence that the $\alpha^{\prime}$ process in
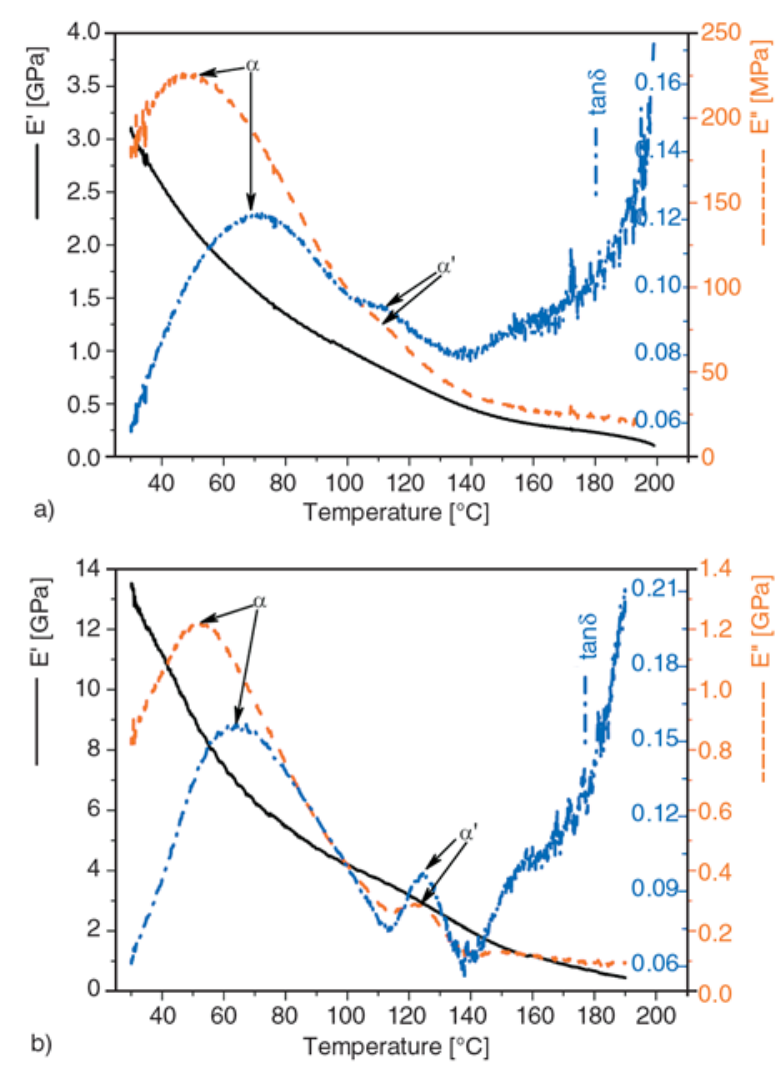

Figure 4. DMA traces of: (a) nylon $66\left(250^{\circ} \mathrm{C} / 5 \mathrm{~h}\right.$, slow cooling) and (b) microcomposite transcrystalline nylon $66\left(250^{\circ} \mathrm{C} / 3 \mathrm{~h}\right.$, quenching $)$ films

the amorphous phase with its associated chain mobility permits the onset of movement of the 010/110 triclinic crystalline planes until the pseudo-hexagonal structure is attained at $T_{B}$. This conclusion is supported by an overview of transition phenomenon in nylon 6 [24], stating that the Brill transition is observed between $80-170^{\circ} \mathrm{C}$ and claiming that this transition cannot be detected usually by DMA, for an especially long annealing period at a high temperature $\left(220^{\circ} \mathrm{C} / 60 \mathrm{~h}\right)$ where an $\alpha^{\prime}$ relaxation at $170^{\circ} \mathrm{C}$ is observed. It is interesting to note that that study [24] claimed that the ' $\alpha$ ' relaxation was associated with the mobility of interfacial amorphous phase which requires crystal mobility as a precursor', which is the reverse order of the causative relation suggested in our study. We maintain, however, that the causative relation aught not to be determined on the basis of a temperature comparison of the $\alpha^{\prime}$ relaxation and $T_{B}$. Rather, it is determined by the relation between the $\alpha^{\prime}$ relaxation and the temperature of the sharp tilt in the $d$ spacings of the $010 / 110$ diffraction which precedes $T_{B}$. 


\section{Conclusions}

The change in the $d$ spacings with temperature of the 010/110 diffraction, which precedes the Brill transition, is characterized by a two step behavior. The step change is characterized by a sharp tilt followed by a steep increase of the $d$ spacing all the way to the Brill transition temperature. It is claimed that this step change reflects a critical free volume change and an onset of segmental chain movement in the amorphous phase. Dynamic mechanical analysis identified in specifically treated nylon 66 and microcomposite samples a prominent $\alpha^{\prime}$ process, which corresponds to this step change. Hence, the observed $\alpha^{\prime}$ relaxation and the Brill transition are taken as counterparts, wherein the occurrence of the first triggers that of the latter.

\section{Acknowledgements}

The authors are indebted to Dr. A. Bytchkov of ESRF, ID11 (MA-464) for his assistance in the synchrotron measurements.

\section{References}

[1] Sperling L. H.: Introduction to physical polymer science. John Wiley and Sons, Hoboken (2006).

[2] Cohn D., Younes H., Marom G.: Amorphous and crystalline morphologies in glycolic acid and lactic acid polymers. Polymer, 28, 2018-2022 (1987).

DOI: $10.1016 / 0032-3861(87) 90035-8$

[3] Tregub A., Harel H., Marom G., Migliaresi C.: The influence of thermal history on the mechanical properties of poly(ether ether ketone) matrix composite materials. Composites Science and Technology, 48, 185-190 (1993). DOI: $10.1016 / 0266-3538(93) 90135-4$

[4] Pegoretti A., Ashkar M., Migliaresi C., Marom G.: Relaxation processes in polyethylene fibre-reinforced polyethylene. Composites Science and Technology, 60, 1181-1189 (2000).

DOI: $\underline{10.1016 / \mathrm{S} 0266-3538(00) 00024-5}$

[5] Alon Y., Marom G.: On the $\beta$ transition in high density polyethylene: The effect of transcrystallinity. Macromolecular Rapid Communication, 25, 13871391 (2004).

DOI: $\underline{10.1002 / \text { marc. } 200400185}$

[6] Brill R.: Behavior of polyamides on heating (in German). Journal für Praktische Chemie, 161, 49-64 (1942).

DOI: 10.1002/prac.19421610104

[7] Brill R.: Relations between the structures of polyamides and silk fibers (in German). Zeitschrift für Physikalische Chemie, B 53, 61-74 (1943).
[8] Sandeman I., Keller A.: Crystallinity studies of polyamides by infrared, specific volume and X-ray methods. Journal of Polymer Science, 19, 401-435 (1956).

DOI: $10.1002 /$ pol.1956.120199303

[9] Jones N. A., Atkins E. D. T., Hill M. J., Cooper S. J., Franco L.: Chain-folded lamellar crystals of aliphatic polyamides. Comparisons between nylons 44,64 , 8 4, 10 4, and 12 4. Macromolecules, 29, 6011-6018 (1996). DOI: $\underline{10.1021 / \mathrm{ma} 9603490}$

[10] Jones N. A., Atkins E. D. T., Hill M. J., Cooper S. J., Franco L.: Chain-folded lamellar crystals of aliphatic polyamides. Investigation of nylons $48,410,412$, 6 10, 612,618 and 8 12. Polymer, 38, 2689-2699 (1997). DOI: $\underline{10.1016 / \mathrm{S} 0032-3861(97) 85603-0}$

[11] Jones N. A., Atkins E. D. T., Hill M. J.: Comparison of structures and behavior on heating of solutiongrown, chain-folded lamellar crystals of 31 even-even nylons. Macromolecules, 33, 2642-2650 (2000). DOI: $10.1021 / \mathrm{ma} 9919559$

[12] Li W., Huang Y., Zhang G., Yan D.: Investigation on Brill transition of nylons $6 / 16,4 / 16$ and $2 / 16$ by variable-temperature WAXD and FTIR. Polymer International, 53, 1905-1908 (2003). DOI: $\underline{10.1002 / p i .1267}$

[13] Cui X., Yan D.: Preparation, characterization and crystalline transitions of odd-even polyamides 11,12 and 11,10. European Polymer Journal, 41, 863-870 (2005). DOI: 10.1016/j.eurpolymj.2004.10.045

[14] Feldman A. Y., Wachtel E., Vaughan G. B. M., Weinberg A., Marom G.: The Brill transition in transcrystalline nylon-66. Macromolecules, 39, 4455-4459 (2006). DOI: $\underline{10.1021 / \mathrm{ma060487h}}$

[15] Murthy N. S., Wang Z-G., Hsiao B. S.: Interactions between crystalline and amorphous domains in semicrystalline polymers: Small-angle X-ray scattering studies of the Brill transition in nylon 6,6. Macromolecules, 32, 5594-5599 (1999). DOI: $10.1021 / \mathrm{ma} 990475 \mathrm{e}$

[16] Klein N., Marom G., Pegoretti A., Migliaresi C.: Determining the role of interfacial transcrystallinity in composite materials by dynamic mechanical thermal analysis. Composites, 26, 707-712 (1995). DOI: 10.1016/0010-4361(95)91137-T

[17] Nuriel H., Kozlovich N., Feldman Y., Marom G.: The dielectric properties of nylon 66/aramid fibre microcomposites in the presence of transcrystallinity. Composites Part A: Applied Science and Manufacturing, 31, 69-78 (2000). DOI: $\underline{10.1016 / \mathrm{S} 1359-835 X(99) 00050-0}$

[18] Nuriel H., Klein N., Marom G.: The effect of the transcrystalline layer on the mechanical properties in the fibre direction of composite materials. Composites Science and Technology, 59, 1685-1690 (1999). DOI: $\underline{10.1016 / \mathrm{S} 0266-3538(99) 00026-3}$ 
[19] Takayanagi M., Harima H., Iwata Y.: Viscoelastic behavior of polymer blends and its comparison with model experiments. Reports on Progress in Polymer Physics (in Japanese), 6, 121-125 (1963).

[20] McCrum N. G., Read B. E., Williams G.: Anelastic and dielectric effects in polymeric solids. John Wiley and Sons, London, 478-500 (1967).

[21] Feldman A., Gonzalez M. F., Wachtel E., Moret M. P., Marom G.: Transcrystallinity in aramid and carbon fiber reinforced nylon 66: Determining the crystallographic structure by synchrotron X-ray micro diffraction. Polymer, 45, 7239-7245 (2004). DOI: $10.1016 /$ j.polymer.2004.08.027
[22] Wagner H. D., Rubins M., Marom G.: The significance of microcomposites as experimental models. Polymer Composites, 12, 233-236 (1991).

DOI: $10.1002 /$ pc.750120404

[23] Klein N., Wachtel E., Marom G.: The microstructure of nylon 66 transcrystalline layers in carbon and aramid fibre reinforced composites. Polymer, 37, 5493-5498 (1996).

DOI: $10.1016 / \mathrm{S} 0032-3861(96) 00361-8$

[24] Khanna Y. P.: Overview of transition phenomenon in nylon 6. Macromolecules, 25, 3298-3300 (1992). DOI: $\underline{10.1021 / \mathrm{ma} 00038 \mathrm{a} 043}$ 